[7] G. M. Foody and D. P. Cox, "Sub-pixel land cover composition estimation using a linear mixture model and fuzzy membership functions," Int. J. Remote Sensing, vol. 15, no. 3, pp. 619-631, 1994.

[8] A. Grace, "Optimization TOOLBOX for use with MATLAB," Math Works, Inc., 1994.

[9] P. E. Gill, W. Murray, and M. H. Wright, Practical Optimization. London, U.K.: Academic, 1981

[10] G. H. Golub and C. F. Van Loan, Matrix Computations, 2nd ed. Baltimore, MD: John Hopkins Univ. Press, 1981, pp. 70-74.

[11] A. R. Harrison, S. J. Hurcom, and M. Taberner, "Examining spectral mixture modeling using in situ spectro-radiometric measurements," in Spatial Data 2000. Nottingham, U.K.: Remote Sensing Soc., 1990, pp. 242-249.

[12] H. M. Horwitz et al., "Estimating the proportion of objects within a single resolution element of a multi-spectral scanner," Univ. Michigan, Ann Arbor, NASA Contract NAS-9-9784.

[13] J. T. Kent and K. V. Mardia, "Spatial classification using fuzzy membership models," IEEE Trans. Pattern Anal. Machine Intell., vol. PAMI-10, pp. 659-671, May 1988.

[14] S. E. Marsh et al., "Resolving the percentage of component terrains within single resolution elements," Photogramm. Eng. Remote Sensing, vol. 46, pp. 1079-1086, Aug. 1980.

[15] N. A. Quarmby et al., "Linear mixture modeling applied to AVHRR data for crop area estimation," Int. J. Remote Sensing, vol. 13, no. 3, pp. 415-425, 1992.

[16] J. J. Settle and N. A. Drake, "Linear mixing and the estimation of ground cover proportions," Int. J. Remote Sensing, vol. 14, no. 6, pp. 1159-1177, 1993.

[17] M. O. Smith et al., "Calibration of AIS images using the surface as a reference," in Proc. 3rd Airborne Image Spectrometer Data Anal. Workshop, 1987.

\section{Response to Comments on, "An Analysis of a Staked Dipole Probe on a Lossy Earth Plane Using the Finite-Difference Time-Domain Method"}

\author{
James R. Wait
}

There are two items that I need to respond to. The first deals with the basis of my expression for the effective complex area of a horizontal electric dipole (HED) over a layered conductive half-space. The second related item deals with a basic flaw in their estimate of the error when the HED is lifted relative to the air/earth interface. In both cases, we orient the HED to have maximum response.

\section{A. Effective Area of Loop for Stratified Earth Model}

Let $E_{v}$ be the vertical electric field in air for a distant very low frequency (VLF) transmitter. Then

$$
E_{v} \cong \hat{n}_{o} H_{h}
$$

where $H_{h}$ is the horizontal magnetic field and $\hat{n}_{o}=j \mu_{o} \omega / j k=$ $120 \pi$. The voltage $v$ induced in the equivalent loop of area $A$ is

$$
v=j \mu_{o} \omega A H_{h} .
$$

But for the HED of effective length $L$

$$
v=E_{h} L
$$

Manuscript received February 17, 1998; revised May 21, 1998.

J. R. Wait, deceased, was with the University of Arizona, Tucson, AZ 85719-3848 USA.

Publisher Item Identifier S 0196-2892(99)00472-6. where $E_{h}=W E_{v}$ in terms of the wave tilt $W$. Then, on equating (2) and (3), we get $A \cong W L / j k$, as given in my comments [2].

\section{B. Tilt Corrections for Staked and Unstaked Dipoles}

Thiel and Mittra ( $\mathrm{T}$ and $\mathrm{M}$ ) assert that the total effective area of their HED of length $L$, raised to a mean height $t$ above the homogeneous half-space, is given by

$$
A_{\text {total }} \cong L / \gamma+L / t=(L / \gamma)[1+\gamma t]
$$

This is equivalent to writing

$$
A_{\text {total }} \cong A(1+\gamma t)
$$

where $\gamma t=(1+j) t / \delta$ in terms of the skin depth of the lower halfspace. As $\mathrm{T}$ and $\mathrm{M}$ indicate in their example, if $|\gamma t|$ or $t / \delta<<1$, the correction to the raised HED is negligible. But they are really dealing with the staked HED. Only in that case, does the correction result from the voltage induced in a closed loop of area $L X t$.

When the elevated HED is not terminated, the correction is quite different. Now the critical parameter is the mean tilt rather than the mean height. Again, for the distant VLF transmitter, the relatively large vertical electric field $E_{v}$ will induce a secondary voltage in the tilted HED, which is approximately $E_{v} L \Theta$, where $\Theta$ is the tilt angle relative to the air/earth interface. In this case

$$
A_{\text {total }} \cong A[1+(\Theta / W)]
$$

where now the correction $\Theta / W$ can be very significant if the tilt angle $\Theta$ is comparable with or greater than the magnitude of the wave tilt $W$. It is interesting to compare the corrections for staked and unstaked cases. The relevant ratio is $\left|(\gamma t) /\left[\Theta W^{-1}\right]\right| \cong k t / \Theta \cong k L$. For $L=10 \mathrm{~m}$, as in the example by $\mathrm{T}$ and $\mathrm{M}$, and for a frequency of $20 \mathrm{kHz}$, this ratio is $4 \times 10^{-3}$. Thus, for this tilted dipole (with zero height at one end to $2 t$ at the other end), the correction for the unstaked case is 250 times that for the staked case.

The key results given by (5) and (6), while based on a highly idealized model, are consistent with the more general analysis in [3].

Of course, I well appreciate that there are many instances in which the unstaked or unterminated horizontal dipoles are to be preferred, as $\mathrm{T}$ and $\mathrm{M}$ point out, but there are also arguments on the other side of the ledger.

\section{REFERENCES}

[1] D. V. Thiel and R. Mittra, "An analysis of a staked dipole probe on a lossy earth plane using the finite-difference time-domain method, "IEEE Trans. Geosci. Remote Sensing, vol. 35, pp. 1357-1362, Sept. 1997.

[2] J. R. Wait, "Horizontal electric field sensors—-staked or not," this issue, p. 312 .

[3] _ , "On measuring the horizontal electric field just above earth's surface," IEEE Trans. Instrum. Meas., vol. 43, pp. 481-483, June 1994. 


\section{Reply to Comments by James R. Wait}

David V. Thiel and Raj Mittra

We are happy with the additional analysis given by Wait in his latest comments on our paper [1].

It may be that Wait's concern for the contaminating field components for horizontal electric field measurements has resulted from the very significant difficulties that we have with making electric field wave tilt measurements in air just above ground. We certainly agree

Manuscript received November 26, 1997; revised January 7, 1998.

D. V. Thiel is with the School of Microelectronic Engineering, Griffith University, 4111 Queensland, Australia (e-mail: dthiel@me.gu.edu.au).

R. Mittra is with the Department of Electrical Engineering, Pennsylvania State University, University Park, PA 16802 USA (e-mail: r1mece@engr.psu.edu).

Publisher Item Identifier S 0196-2892(99)00329-0. that making such wave tilt measurements is very difficult. However, our extensive experimental work with the insulated dipole resting on the surface of the earth [2]-[4], in addition to the numerical modeling results that were presented in our original paper [1], has given us confidence that this method of approach is very satisfactory.

\section{REFERENCES}

[1] D. V. Thiel and R. Mittra, "An analysis of a staked dipole probe on a lossy earth plane using the finite-difference time-domain method," IEEE Trans. Geosci. Remote Sensing, vol. 35, pp. 1357-1362, Sept. 1997.

[2] D. V. Thiel and F. B. Neall, "VLF surface impedance measurements in the Antarctic, " J. Glaciol., vol. 35, pp. 197-200, 1989.

[3] D. V. Thiel, "Surface impedance changes in the vicinity of an abrupt lateral boundary at the earth's surface," IEEE Trans. Geosci. Remote Sensing, vol. GE-28, pp. 500-502, Mar. 1990.

[4] M. Sitepu and D. V. Thiel, "The surface impedance anomaly resulting from a subsurface infinitely long conductive wire," IEEE Trans. Geosci. Remote Sensing, vol. GE-29, pp. 314-320, Mar. 1991. 